\title{
Identificación de atracciones urbanas centrales mediante seguimiento GPS y análisis de redes
}

\author{
Identification of central urban attractions based \\ on GPS tracking data and network analysis
}

\author{
Ibon Aranburu Amiano \\ ibon.aranburu@ehu.eus \\ Departamento de Organización de Empresas \\ UPV-EHU (España)
}

Beatriz Plaza Inchausti

beatriz.plaza@ehu.es

Departamento de Economía Aplicada

UPV-EHU (España)

\section{Marisol Esteban Galarza}

marisol.esteban@ehu.es

Departamento de Geografía

UPV-EHU (España)

\section{Resumen}

Este estudio presenta una metodología aplicable en la identificación de atracciones turísticas centrales en entornos urbanos mediante el uso combinado de datos GPS y análisis de redes de atracciones visitadas por los turistas. La identificación de las atracciones centrales es fundamental para los gestores de una ciudad, tanto a la hora de planificar las instalaciones y servicios urbanos, - gestionar los recursos municipales, como localizar nuevas atracciones o captar todos los 
beneficios potenciales de los mismos. El primer paso de la metodología propuesta es la detección de las atracciones visitadas mediante el análisis de datos GPS. A partir de este conjunto de datos GPS se construye una red cuyos nodos son las atracciones visitadas y posteriormente se realiza el análisis de redes correspondiente. El estudio empírico se ha llevado a cabo en la ciudad de Bilbao, destino turístico que ha obtenido fama internacional gracias al Museo Guggenheim. Sorprendentemente, nuestra metodología conduce a resultados inesperados: mientras que los contenidos de las redes sociales (por ejemplo, TripAdvisor) y los expertos (agentes turísticos) señalan al Guggenheim como el principal activo turístico, en realidad resulta ser el Casco Viejo el lugar más visitado de Bilbao según el comportamiento espacial real detectado por nuestro método. Este enfoque metodológico puede servir para tomar decisiones más adaptadas y definir mejores políticas en materia de planificación y gestión urbana.

Palabras clave: centralidad; geografía urbana; planificación urbana; atracciones centrales; comportamiento espacial humano.

\begin{abstract}
This study introduces a useful methodology to identify central urban tourism attractions based on the combination of GPS tracking data and the Network Analysis of visited attractions derived from GPS data. Identifying central attractions becomes critical for city managers when it comes to planning urban facilities, managing municipal resources, locating new attractions or capturing all the potential returns. The first step of the proposed methodology is the detection of visited attractions based on GPS tracking data analysis. Then from this GPS data set a network of visited attractions is built in order to carry out a network analysis. The empirical study is performed for the city of Bilbao, a tourism destination made famous by the Guggenheim Museum. Surprisingly, our methodology leads to unexpected results: while social media content (e.g. TripAdvisor) and experts (tourism agents) point to the Guggenheim as the main tourism asset, in fact it turns out to be the Old Town the most visited place in Bilbao according to real spatial behavior detected by our method. This methodological approach can be valuable for performing decisions that are more accurate and better policies concerning urban planning and management.
\end{abstract}

Keywords: centrality; urban geography; urban planning; central attractions; human spatial behavior. 


\section{Introducción}

Uno de los principales hitos del desarrollo urbano de los últimos años es el considerable aumento del número de visitantes urbanos y su movilidad (Le-Klähn, 2016). Este hecho implica un reto para los gestores de las ciudades y las políticas urbanas, ya que las ciudades tienen que hacer frente a este aumento de la demanda ofreciendo recursos y servicios en consonancia, como por ejemplo ofreciendo servicios de transporte con una mayor frecuencia. En este contexto, la identificación de las atracciones centrales de una ciudad se ha convertido en una cuestión crítica ya que permite alcanzar una planificación y una gestión urbana más eficiente y sostenible. Se entiende por atracciones centrales, aquellos lugares de interés más visitados por los turistas y que están mejor interconectados con las demás atracciones.

La centralidad de las atracciones urbanas es un concepto multidimensional y se puede medir de diversas formas (popularidad, centralidad geográfica, centralidad de red, efectos de aglomeración). Las mediciones de centralidad presentadas en este estudio se basan en el análisis de la topología de red construida a partir del consumo espacial (Scott, 2000). En esta investigación se adoptan cuatro índices de centralidad de red (Freeman, 1978), a saber: el número de conexiones que tiene la atracción; la accesibilidad de una atracción; la distancia media a las demás atracciones; y la influencia sobre las atracciones urbanas circundantes (ver Tabla 1). Estos índices de centralidad son clave para evaluar las atracciones urbanas.

Para realizar el análisis de la centralidad de red, previamente es necesario construir una red de atracciones. En este estudio, la red de atracciones construida se basa en el comportamiento de la movilidad de los visitantes y considera que existe una conexión entre dos atracciones si una persona visita ambas. La comprensión de la movilidad de los visitantes es una cuestión clave para el desarrollo urbano y económico, ya que permite geolocalizar los lugares en los que se desarrollan las actividades humanas y permite identificar las atracciones centrales. La investigación del comportamiento espacial de los visitantes está relacionada con el problema fundamental en la geografía y la economía geográfica de la ubicación de las actividades y su distribución espacial (Barthélemy, 2011).

Los resultados obtenidos a partir de esta metodología son diferentes a los de otras clasificaciones de atracciones, como las basadas en el contenido de las redes sociales (por ejemplo, las opiniones de TripAdvisor, las fotos de Instagram, etc.), los expertos (agentes turísticos) o el conocimiento colectivo de la población local. En nuestro caso, el ranking de centralidad se mide teniendo en cuenta el comportamiento espacial de los visitantes y la topología de la red de 
atracciones visitadas, lo que refleja qué atracciones son las más visitadas y cuál es la interacción entre ellas.

Cabe destacar que esta metodología no sólo es válida para atracciones de primer nivel sino también para atracciones de nivel inferior o no tan conocidas. Estas atracciones normalmente no aparecen en los rankings asociados a las redes sociales, pero lógicamente también tienen que ser planificadas por los gestores urbanos. Teniendo en cuenta esto último, esta metodología también permite detectar nuevas atracciones o puntos de interés, que quizás no forman parte de las guías turísticas de la ciudad. Una vez dicho esto, habría que matizar que no todas las redes sociales funcionan por rankings y que también es posible hacer un seguimiento de las atracciones de cualquier nivel analizando los datos de geolocalización de los contenidos compartidos en las redes sociales (Zornoza Gallego \& Salom Carrasco, 2018).

En definitiva, el objetivo de este estudio es responder a la necesidad de los gestores de la ciudad y a los responsables de la toma de decisiones, presentando una metodología útil que proporcione información sobre las atracciones centrales basada en el consumo espacial de los visitantes.

\section{Estado de la cuestión}

\subsection{Las atracciones urbanas como objeto de estudio}

Las atracciones de la ciudad, entendidas como un lugar de interés tanto para los visitantes como para los propios habitantes de la ciudad, son la base para la creación de una ciudad atractiva y el éxito de un destino urbano. Es reconocido que las atracciones urbanas pueden ayudar a definir la propia ciudad, pudiendo llegar a formar parte de su propia identidad, diferenciándola de los demás destinos y obteniendo así una ventaja competitiva en el mercado turístico (Richards, 2010). Además, pueden convertirse en polos de atracción dentro de las rutas turísticas. Así, algunos autores (Kourtit, Nijkamp \& Partridge, 2013; Richards, 2011, 2014) reivindican el potencial que tiene la creación de nuevas atracciones en el desarrollo urbano, desde una perspectiva tanto socioeconómica como cultural. De hecho, algunos autores sostienen que las atracciones de las ciudades/localidades son uno de los factores más importantes para la organización de las redes turísticas (Urtasun \& Gutiérrez, 2006).

Aquellos destinos que dispongan de un patrimonio valioso y/o abundante disponen de una ventaja competitiva sobre los demás, ya que son capaces de ofertar un producto y posicionarse en el mercado de forma natural y con mucho menos esfuerzo. Para estos destinos el turismo cultural se presenta como una oportunidad clara de desarrollo y crecimiento (Silberberg, 1995). En 
cualquier caso, con el objeto de ser sostenibles todos los destinos requerirán de una planificación integral del destino y una gestión de sus productos turísticos y de sus flujos de demanda (Le-Klähn, 2016).

La determinación del centro o centros de la ciudad es una problemática mayor, abordada por la geografía urbana desde sus inicios, y principalmente desde que surgieran los distritos comerciales o de negocios, más conocidos en inglés como Central Business District, CBD (Hartman, 1950; Jayasinghe, Sano \& Rattanaporn, 2017; Yu, Ai \& Shao, 2015). En este sentido, la identificación de los elementos urbanos (atracciones culturales, instalaciones públicas, servicios, etc.) con mayor centralidad es clave ya que tienen implicaciones importantes en el desarrollo urbano (Capello \& Perucca, 2017; Sacco, Ferilli \& Blessi, 2014; Throsby, 2017).

Un aspecto clave a la hora de analizar las atracciones en una ciudad reside en la interacción de los visitantes con los distintos espacios/lugares turísticos (museos, restaurantes, monumentos, etc.) y estudiar cómo estos recursos se relacionan entre sí (Leung et al., 2012). En este sentido es clave medir la centralidad de estos recursos y cuál es su nivel de atracción (Aranburu, Plaza \& Esteban, 2016). Para llevar a cabo esta tarea, el análisis de redes es de gran ayuda ya que permite analizar las interacciones espaciales entre los distintos recursos turísticos y medir su centralidad. Además, permite analizar otras características de centralidad de los nodos de la red como su accesibilidad, cercanía e intermediación. Por su parte, algunos autores (Liu, Huang \& Fu, 2017) van más allá y no sólo identifican las redes de atracciones existentes producidas por los movimientos de turistas, sino que también intentan establecer vínculos causales entre las atracciones relevantes y los flujos turísticos.

\subsection{Consumo Espacial y Movilidad Urbana}

La movilidad de los turistas y su comportamiento espacio-temporal tiene una profunda implicación en los destinos turísticos en términos generales y más concretamente en aspectos como las infraestructuras de transporte, el desarrollo de productos turísticos, las estrategias de marketing, la visibilidad comercial de la industria turística y la gestión del impacto social, cultural y medioambiental del turismo. Así, el deseo de comprender la movilidad de los turistas ha sido de interés para los investigadores desde hace varios años (Dietvorst \& Ashworth, 1995; Shaw, Agarwal \& Bull, 2000).

Los turistas utilizan distintos modos de transporte a la hora de desplazarse dentro de un destino. En los últimos años, gracias a los proyectos de regeneración urbana desarrollados en múltiples ciudades, se está fomentando la movilidad peatonal de los turistas y los propios habitantes, en aras 
de la sostenibilidad de la ciudad (Hall \& Ram, 2019). Un ejemplo paradigmático de este fenómeno es el caso de Bilbao (Esteban, 1999), cuyo plan de regeneración urbana modificó los patrones de movilidad de manera espectacular, abriendo la ciudad al borde del río y ampliando las rutas peatonales a lo largo de la ribera, aumentando así la transitabilidad y mejorando la calidad de vida de Bilbao.

En un principio, las investigaciones relacionadas se centraron principalmente en el flujo de turistas entre destinos, o en la movilidad de un mercado origen a otras áreas de destino, empleando conceptos como acceso a mercados, estimación de tiempos, distancias etc. Pero la problemática metodológica que conllevan estos estudios ha impulsado a los investigadores a realizar estudios similares en áreas más reducidas, como es el caso de los destinos urbanos (Shoval \& Isaacson, 2009). Esta problemática reside en la propia naturaleza de los estudios de campo basados en encuestas ya que es cuestionable la credibilidad de los datos aportados por los participantes, que a menudo no logran transmitir fielmente sus acciones y/o visitas a las distintas atracciones a lo largo de su viaje.

El turismo urbano se caracteriza por una serie de actividades que realiza el turista en el destino (pasear, hacer compras, comer en un restaurante, visitar museos, etc.). Estas actividades están claramente ligadas al tiempo y al espacio, pero a pesar de ello hasta hace poco no se ha puesto suficiente atención a la movilidad de los turistas dentro de las líneas de investigación de la geografía humana y la investigación turística (Shaw et al., 2000). Este retraso se ha debido principalmente a la complejidad metodológica de los estudios de medición de la movilidad.

A partir del año 2000, el rápido desarrollo y la disponibilidad de dispositivos de seguimiento (tracking) más pequeños, baratos y fiables ha conducido al incremento de las investigaciones en general y en los estudios turísticos en particular. Los dispositivos GPS (Global Positioning System) ofrecen una captura de datos de alta resolución continua e intensiva, en tiempo y en espacio. Así el seguimiento GPS se ha convertido en un método popular para recopilar información relacionada con la movilidad de los turistas y conocer los patrones de consumo del espacio. En la siguiente subsección se puede ver con más detalle algunas características de los dispositivos GPS.

Las primeras investigaciones sobre datos obtenidos por GPS estuvieron orientadas a los estudios de transporte (Bohte \& Maat, 2009; Quiroga \& Bullock, 1998; Wolf, Guensler \& Bachman, 2001). Pero los estudios de la actividad espacial de los turistas no tardaron en llegar, una vez se consiguió fabricar dispositivos GPS más pequeños, ligeros y con mayor sensibilidad (Ashbrook \& Starner, 2003; Axhausen, Schonfelder, Wolf, Oliveira \& Samaga, 2003; Schönfelder, Axhausen, Antille, \& 
Bierlaire, 2002; Timmermans, Arentze \& Joh, 2002). Posteriormente varios autores (Lew \& McKercher, 2006; Mckercher \& Lau, 2008) analizaron los factores que influían en la movilidad del turista, identificando factores relacionados con las características del destino y del turista. Otras investigaciones se han centrado en características específicas en relación al impacto de la actividad espacial (Gschwender, Munizaga \& Simonetti, 2016; McKercher, Shoval, Ng \& Birenboim, 2012; Shen \& Stopher, 2014). Además, numerosos estudios (Grinberger, Shoval, \& McKercher, 2014; Mazimpaka \& Timpf, 2015) han vinculado bases de datos de recursos y atracciones urbanas a los datos del GPS, con el fin de identificar el tipo de actividad que se realiza en cada lugar, de la misma forma que se ha realizado en este trabajo.

En relación a los factores que influyen en la movilidad de los turistas, las variables que se consideran generalmente importantes pueden dividirse en tres tipos de grupos (Shoval \& Isaacson, 2009): El primer grupo de factores estaría relacionado con las características del viaje (duración del viaje, motivo del viaje, número de visitas al destino, organización del viaje, etc.). El segundo grupo se asociaría con las características específicas del turista (país de origen, género, religión, ingresos, educación, tipo de personalidad, etc.). Y se tendría un tercer grupo donde se tendrían en cuenta las variables externas (tiempo meteorológico, transporte, masificación, etc.).

A continuación, se puede apreciar el modelo conceptual desarrollado por el geógrafo alemán Tim Freytag (Freytag, 2002), donde se presenta gráficamente las diversas variables y restricciones espacio-temporales de las actividades turísticas que tienen lugar en un destino durante la visita de un turista.

Figura 1. Variables que afectan a la movilidad en un destino

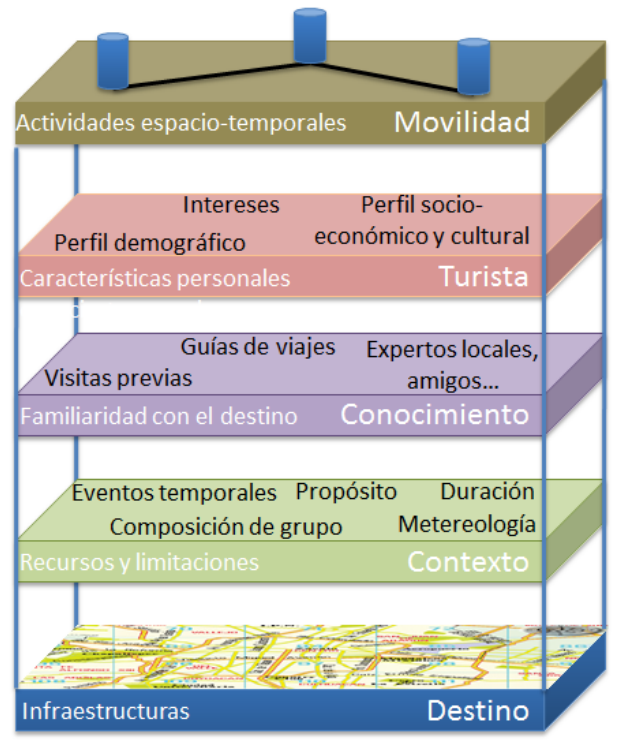

Fuente: representación gráfica basada en Freyłag (2002) (Shoval \& Isaacson, 2009) 
Tal y como se puede observar en la Figura 1, Freytag deconstruye la movilidad espacio-temporal en 4 capas: destino, contexto o visita, conocimiento previo del destino y perfil del turista. En la primera capa de destino se encuentra lo relacionado con la dimensión física del territorio, la orografía y las infraestructuras existentes en el mismo, como la red de transporte. Así, autores como Page (2004) trataron de analizar la relación existente entre la movilidad del turista y la red de transporte del destino. La segunda capa del modelo, pertenece a las variables relacionadas con la propia visita, como la composición del grupo, propósito general de la visita, "time-budget" o tiempo disponible de la visita, etc. Esta última variable ha sido estudiada en profundidad por autores como Pearce (1988) quien afirmaba que el tiempo consumido en una zona específica del destino es el criterio más influyente a la hora de determinar el comportamiento turístico. La tercera capa comprende el conocimiento y la familiarización que tiene el turista con el destino. En esta capa, se pueden considerar aspectos como la utilización de guías o las visitas previas realizadas al destino. Existen diversos estudios que tratan de relacionar el uso que realiza el visitante de la información disponible del destino y la manera en la que consume el mismo (Lew \& McKercher, 2006; McKercher et al., 2012). Por último, en la cuarta capa se incluye el perfil sociodemográfico del turista. Así, una serie de estudios tratan de comprender el efecto que tienen sobre la movilidad el perfil del visitante/turista (Grinberger et al., 2014; Shoval, McKercher, Ng \& Birenboim, 2011; Tchetchik, Fleischer \& Shoval, 2009).

De todas formas, todavía queda lugar para la investigación de los factores que subyacen a la movilidad y a la actividad espacial de los turistas en un entorno urbano, debido a la complejidad de desarrollar un marco teórico completo (Shoval \& Isaacson, 2009).

a) Datos de movilidad

Hasta principios del siglo XXI, el método más común de captura de datos en relación a los patrones espacio-temporales de los turistas ha sido el diario de viaje espacio-tiempo. Este método provee de un registro sistemático de la forma en el que el individuo ocupa su tiempo en el espacio durante un período particular. El diario espacio-tiempo requiere que los sujetos encuestados participen activamente en el proceso de captura de datos, apuntando sus actividades durante todo el experimento. De manera que la credibilidad de los datos es a menudo cuestionable (Murakami \& Wagner, 1999; Wolf et al., 2001).

En la actualidad existen otras posibilidades para medir la movilidad de los turistas, haciendo uso de diversas fuentes de datos: los registros de teléfonos móviles, transacciones con tarjetas de crédito, datos de redes sociales (contenido geoetiquetado: fotografías, opiniones, etc.), registros 
en aplicaciones de Internet, etc. (Abedi, Bhaskar \& Chung, 2014; García-Palomares, Gutiérrez, \& Mínguez, 2015; Hasan, Schneider, Ukkusuri \& González, 2013; Hawelka et al., 2014; Ratti et al., 2010; Zornoza Gallego \& Salom Carrasco, 2018). También es posible obtener información sobre la movilidad humana mediante el análisis de datos GPS de dispositivos integrados en todo tipo de vehículos, como taxis, autobuses, bicicletas, etc. (Yuan, Zheng \& Xie, 2012).

Una de las diferencias existentes entre las fuentes de datos mencionadas es que para la mayoría de ellas no se dispone de series largas y continuas de datos espacio temporales para cada visitante, como ocurre en el caso de los datos de GPS, sino que se dispone de transacciones geolocalizadas en momentos concretos y aleatoriamente distantes en el tiempo. Este último hecho tiene un gran calado a la hora de identificar y analizar las rutas o los recorridos realizados por los visitantes. Aunque siempre es posible determinar el recorrido a partir de geolocalizaciones sucesivas, en este caso la sucesión de transacciones es aleatoria y puede producirse en intervalos de tiempo en el que no sea posible considerar un mismo recorrido. A pesar de estas limitaciones, el análisis de estos datos discretos también permite extraer conocimiento de interés sobre la movilidad de los turistas dentro de un destino. En este trabajo se consideran los datos obtenidos de los dispositivos GPS llevados por los propios visitantes para medir su movilidad.

\section{b) Dispositivos GPS}

El GPS (Global Positioning System) es un sistema global de navegación por satélite (Global Navigation Satellite System, GNSS) que permite conocer la geoposición (latitud, longitud y altitud) del receptor (persona, vehículo, animal, etc.). El GPS fue originalmente concebido como un sistema de navegación militar y su servicio fue totalmente operativo desde 1994 (Kaplan, 1996), Pero no fue hasta mayo de 2000 que el sistema se abrió al público en general y a las aplicaciones comerciales en todo el mundo. Así, a finales de los años noventa aparecen los primeros estudios usando tecnología GPS, Quiroga and Bullock (1998) realizaron un estudio sobre la duración del viaje integrando las tecnologías GPS y SIG, Sistema de Información Geográfica. Hay que mencionar que los SIG son sistemas informáticos que se dedican a capturar, almacenar, verificar, integrar, manipular, analizar y mostrar datos espaciales. Su principal objetivo consiste en añadir valor a los datos espaciales permitiendo que se organicen y visualicen de forma eficiente, integrándolos con otros datos, permitiendo su análisis y la creación de nuevos datos (Lau \& McKercher, 2006). Por su parte, Murakami and Wagner (1999) analizaron cómo la tecnología GPS mejora los métodos de estudio de viaje utilizados anteriormente (encuestas, diario de viaje, etc.). 
Desde que se completó el sistema GPS su desarrollo ha sido imparable. Uno de los hitos que ha generalizado el uso del GPS y el uso de servicios georeferenciados ha sido la aparición de los smartphones o teléfonos inteligentes (que disponen entre otros sensores de receptores GPS), ya que son dispositivos que han conseguido una gran penetración de mercado en poco tiempo y además por lo general se hace un uso intensivo de ellos.

En relación a los dispositivos GPS hay que señalar que están construidos mediante sensores/receptores de señales de satélite que pueden integrarse en todo tipo de objetos. En esta investigación se ha hecho uso de los GPS básicos o GPS data loggers, que se describen más detalladamente en la metodología en el apartado de obtención de datos.

Otro aspecto a tener en cuenta es la transmisión de los datos GPS, una vez que han sido registrados por los dispositivos. Existen dos opciones principales, una es la conexión directa (cable USB, bluetooth, etc.) con un PC para la posterior descarga y consulta de los datos haciendo uso de un software especializado instalado en el ordenador. La segunda opción de transmisión consiste en transmitir en tiempo real los datos de posicionamiento mediante sistemas inalámbricos (sistemas de radio frecuencia o sistemas de telefonía móvil) a un servidor web externo, donde se irán almacenando en una base de datos para su posterior explotación. Este último sería el caso de las app de los teléfonos móviles. Los GPS básicos y los navegadores de mano utilizan principalmente la primera opción de transmisión. Los navegadores GPS generalmente no graban el recorrido ni se conectan a un PC, y en teoría son sistemas cerrados. Los GPS integrados, al estar incorporados principalmente a dispositivos móviles, utilizan la segunda opción de transmisión. Hay que tener en cuenta que para el caso de la comunicación mediante telefonía móvil, es necesario que el dispositivo disponga de una tarjeta SIM.

c) Movilidad y ánálisis de redes

Respecto a la literatura relacionada, el seguimiento GPS se ha convertido en un método popular para recopilar información vinculada con la movilidad humana y el consumo del espacio. Sin embargo, la mayoría de los estudios que analizan la movilidad se han limitado a la visualización y análisis de datos GPS, sin combinar un análisis de redes (Galí Espelt \& Donaire Benito, 2018; Gschwender et al., 2016; McKercher et al., 2012; Shen \& Stopher, 2014).

En los últimos años han aparecido algunos estudios que combinan el análisis de redes con los datos de movilidad. En cuanto al tipo de datos de movilidad utilizados en estos estudios, se pueden distinguir cuatro grupos principales: en primer lugar, los estudios basados en datos de tarjetas de transporte (Soh et al., 2010; Zhong et al., 2017) cuyo objetivo es detectar centros de 
transporte y estaciones centrales de transporte, analizando el sistema de tránsito o circulación y asociándolo con los espacios urbanos. En segundo lugar, las investigaciones que utilizan diarios de viaje o encuestas obtenidas de sitios web de redes sociales (Leung et al., 2012) o de agentes turísticos oficiales (Stienmetz \& Fesenmaier, 2015) con el fin de identificar las atracciones turísticas y los patrones de movilidad turística. Tercero, los estudios basados en datos de teléfonos móviles (Ratti et al., 2010), muy útil a la hora de fijar los límites de las ciudades o de identificar las estructuras de agrupación. Y cuarto, los estudios que analizan los datos GPS (Modsching, Kramer, Hagen, \& Gretzel, 2008), al igual que en este trabajo, para detectar las atracciones de interés y el comportamiento espacial de los visitantes. Algunas de ellas se aplican con un alcance diferente en áreas como los parques naturales o recreativos (Taczanowska et al., 2014) y con otros objetivos (Wang, Pedreschi, Song, Giannotti \& Barabasi, 2011). Hay que señalar que el uso de datos GPS tiene algunas ventajas sobre otros tipos de datos de movilidad. Por un lado, representan datos reales de los movimientos de los visitantes, frente a los diarios de viaje o los datos de las redes sociales. Por otra parte, según la frecuencia de la recogida de datos del GPS, los resultados podrían explicar la movilidad con más detalle que otros tipos de datos.

En cuanto al análisis de redes realizado en estos estudios, se pueden identificar dos tipos principales: grafos unidireccionales no ponderados y grafos direccionales ponderados. El primer enfoque es adecuado para el análisis topológico de redes espaciales en términos de accesibilidad basados en las propiedades de red (Demšar, Špatenková \& Virrantaus, 2008; Liu et al., 2017). El segundo tipo es apropiado para analizar la dirección del flujo de visitantes de una atracción a otra y el número de turistas en cada flujo, detectando las rutas principales (Leung et al., 2012; Modsching et al., 2008; Zhong et al., 2017). En esta investigación se ha hecho uso del primer enfoque utilizando grafos unidireccionales no ponderados. Esta elección se justifica porque los grafos unidireccionales no ponderados se presentan como la solución más sencilla que permite realizar el análisis de la topología de red y permite saber si existe interacción entre las atracciones.

\section{Metodología}

A continuación, se presenta la metodología desarrollada para identificar las atracciones urbanas centrales a partir del comportamiento espacial de los turistas. Esta metodología se basa en dos tareas principales: 1) Extracción de las atracciones visitadas a partir del análisis de datos GPS; y 2) Identificación de las atracciones centrales a través del análisis de redes que conforman estas atracciones visitadas. 
La Figura 2 muestra el diagrama de procesos seguido en la metodología, incluyendo las herramientas de software utilizadas para realizar las tareas y obtener los resultados. El proceso comienza con los datos GPS obtenidos mediante los dispositivos GPS, que son el conjunto de datos de entrada y se encuentran almacenados en una base de datos PostgreSQL. Luego se filtran los datos, haciendo un preprocesamiento de los datos GPS. Posteriormente, se detectan los puntos de parada utilizando secuencias de comandos SQL y estos puntos de parada se vinculan a la base de datos de atracciones con el objeto de extraer las atracciones visitadas. A partir de las atracciones visitadas, se construye un grafo no ponderado y no dirigido que es la base para el análisis topológico de redes que se explica más adelante. El análisis de redes incluye la obtención de las propiedades básicas del grafo, calculando varias medidas de centralidad que servirán para identificar las atracciones centrales. Cada uno de estos pasos se explica más detalladamente a continuación.

Figura 2. Diagrama de flujo de la metodología

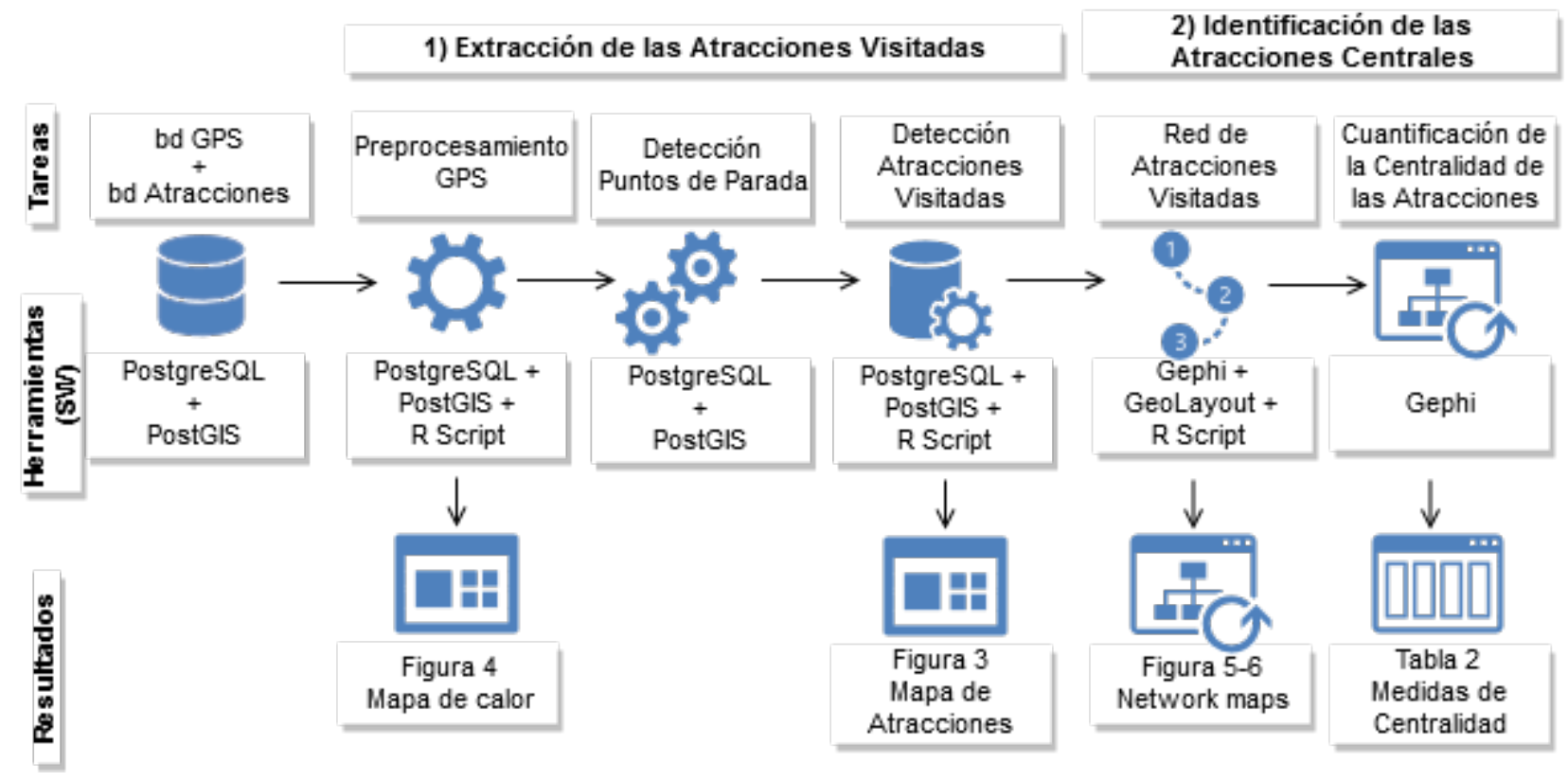

Fuente: elaboración propia usando draw.io

\subsection{Extracción de las atracciones visitadas}

Los datos de tracking GPS se componen de puntos geolocalizados sucesivos, conocidos como waypoints que forman trayectorias. Estos waypoints, más allá de su geolocalización, no representan ningún lugar específico. Por esta razón, se requiere de un análisis de los datos GPS para poder asociar estos waypoints con lugares específicos y extraer así las atracciones visitadas. De esta forma, la identificación de las atracciones visitadas se basa en el análisis de datos GPS, que consta 
a su vez de tres pasos: 1) Preprocesamiento de los trackings GPS; 2) Detección de los puntos de parada; y 3) Extracción de las atracciones visitadas.

a) Preprocesamiento de los trackings GPS

Los métodos de recopilación de datos GPS generalmente contienen errores debido a que los satélites no proveen de una cobertura completa (Shen \& Stopher, 2014). Por este motivo los datos GPS deben ser limpiados antes de ser analizados, eliminando cualquier dato no válido, sea debido a la mala calidad de la señal, sea por velocidades incoherentes, etc. (Stopher, Jiang \& FitzGerald, 2005). Además, los datos GPS relacionados con una estancia prolongada, como la estancia en un hotel o restaurante, conviene agregarlos ya que concentran múltiples puntos geolocalizados en el mismo lugar o área. También conviene señalar que la precisión media de geoposicionamiento de los dispositivos GPS estándares en condiciones ideales oscila entre cinco y diez metros (Schuessler \& Axhausen, 2009), pudiendo mejorarse dicha precisión mediante la utilización de sistemas de aumentación (como WAAS, Wide Area Augmentation Systems) y técnicas de diferenciación (DGPS, Differential GPS). Además, hay que tener en cuenta que la señal GPS puede verse afectada en los entornos urbanos densos, desviación generalmente conocida como efectos multitrayecto. Pero en nuestro caso no ha supuesto un problema ya que el caso de estudio analizado (Bilbao) no es tan denso y en principio se han analizado las atracciones visitadas (lugares específicos) y no el camino seguido de una atracción a otra.

b) Detección de los puntos de parada

Con objeto de extraer las visitas a atracciones urbanas a partir de los trackings GPS es necesario identificar los puntos de parada. Para esta tarea, es necesario aplicar un algoritmo que detecte las paradas efectuadas a partir de los datos del GPS (Fu, Tian, Xu \& Qiao, 2016; Montoliu, Blom \& Gatica-Perez, 2013; Schuessler \& Axhausen, 2009; Xiang, Gao \& Wu, 2016). El algoritmo aplicado considera que existe un punto de parada cuando un visitante permanece en un lugar (umbral de distancia) durante un tiempo mínimo (umbral de tiempo). Por esta razón, no se consideran paradas muy cortas, como paradas en semáforos, paradas cortas para descansar, etc. Finalmente, a la hora de establecer los puntos de parada, también se tiene en cuenta la pérdida de señal, ya que este hecho ocurre generalmente cuando las personas están dentro de un edificio. En este estudio se ha empleado como umbral de distancia un valor inicial de 20 metros y como umbral de tiempo un valor de 10 minutos.

c) Extracción de las atracciones visitadas 
Para detectar las atracciones visitadas, hay que vincular la base de datos de trackings GPS con una base de datos de atracciones georreferenciada. De esta manera, si el visitante pasa más de un tiempo predeterminado dentro del área de influencia de la atracción, se entiende que el visitante está visitando la atracción. Como muchas atracciones no tienen límites exactos (por ejemplo, la Torre Eiffel), se puede definir un área de influencia para cada atracción de acuerdo con su categoría (monumentos, museos, etc.), similar a como lo hicieron Bohte \& Maat (2009). Sin embargo, la definición de un valor fijo para el área de influencia se presenta insuficiente y requiere realizar algunas pruebas y simulaciones variando su radio para asignar una atracción a cada punto de parada detectado. En caso de que el punto de parada se encuentre dentro del área de influencia de más de una atracción, se seleccionará la atracción más cercana (Bohte \& Maat, 2009; Montoliu et al., 2013; Peng \& Huang, 2017). Esta última solución puede resultar simplista, de manera que a la hora de decantarse por una atracción habría que tener en cuenta otros parámetros como la hora de la parada, la duración total de la parada o la importancia de la atracción. También podría ocurrir que un punto de parada no coincida con ninguna atracción de la base de datos. En ese caso, puede ser conveniente indagar más sobre este punto de parada y evaluar si es conveniente añadirlo a la base de datos como una nueva atracción.

\subsection{Identificación de las atracciones centrales}

Una vez que se conocen las atracciones visitadas, la identificación de las atracciones centrales se puede realizar siguiendo estos dos pasos: 1) Elaboración de la red de atracciones visitadas; y 2) Cuantificación de la importancia de los nodos de la red por medio de las medidas de centralidad.

a) Elaboración de la red de atracciones visitadas

Tras detectar las atracciones visitadas, se crea una red para analizar la interacción espacial de las atracciones urbanas y cuantificar su centralidad. Una red se compone principalmente de un conjunto de nodos y arcos. Los nodos representan entidades individuales dentro de la red, en nuestro caso atracciones, y los enlaces representan relaciones entre los nodos. En la teoría de grafos, una red se modela mediante una matriz de adyacencia. La matriz de adyacencia de las atracciones visitadas se construye codificando cada celda con un 1 cuando un visitante ha visitado las dos atracciones y en caso contrario se codifica con un 0 . Por ejemplo, la Figura 3 ilustra la matriz resultante para un turista que visita las atracciones B, C y E. Esta metodología tiene una limitación que conviene señalar y consiste en que no refleja la secuencia de visita de las atracciones (Stienmetz \& Fesenmaier, 2015). 
Figura 3. Ejemplo de matriz de la red de atracciones visitadas

Matriz de Adyacencia

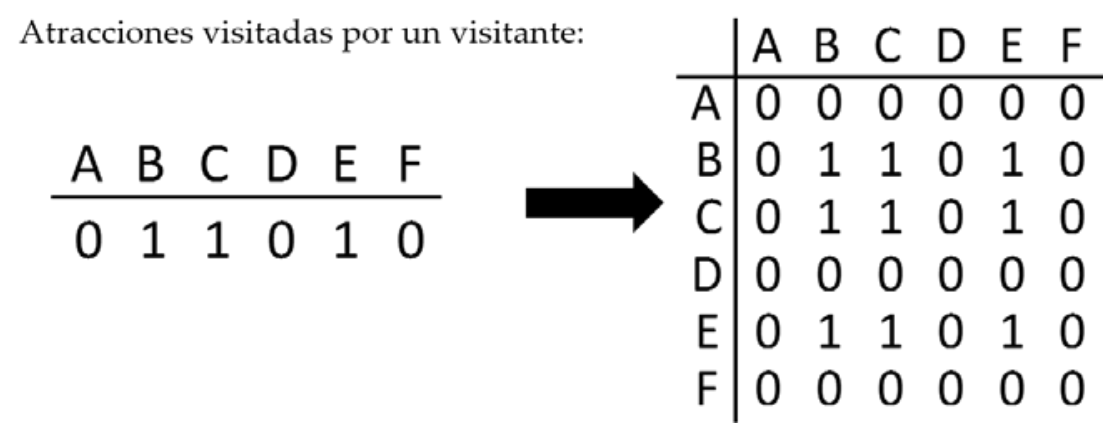

Fuente: elaboración propia

b) Cuantificación de la centralidad de los nodos de la red

Para cuantificar la importancia de los nodos, se estiman cuatro índices de centralidad (gradodegree, intermediación-betweenness, cercanía-closeness, y vectores propios-eigenvectors). Según lo definido por Freeman (1978), la centralidad de grado es un recuento de la cantidad de conexiones directas de un nodo (ver Tabla 1). La centralidad de intermediación mide la accesibilidad de un nodo, teniendo en cuenta si el nodo está ubicado en la ruta más corta entre los nodos restantes. Con respecto a la centralidad de proximidad, esta es una medida de distancia entre un nodo y todos los demás nodos. Por último, la centralidad eigenvector es una medida de la importancia o influencia de un nodo en una red, y se basa en la idea de que un nodo es más central si está relacionado con nodos que son a su vez centrales (Ruhnau, 2000). Al calcular el vector propio, se debe tener en cuenta que cada nodo conectado tiene una ponderación diferente, dependiendo de sus conexiones.

Otro índice interesante de la red es la longitud media de ruta/paso (average path length), calculada entre pares de nodos alcanzables. Este es el número promedio de pasos a lo largo de las rutas más cortas para todos los posibles pares de nodos de la red y representa una medida de la eficiencia del transporte en una red. 
Tabla 1. Medidas de Centralidad de Freeman y Eigenvector de Bonacich.

\begin{tabular}{|c|c|c|}
\hline Centralidad & Descripción & Ecuación \\
\hline Grado & Número de conexiones de un nodo (i) & $\begin{array}{l}\qquad \mathrm{C}_{\mathrm{D}}(\mathrm{i})=\sum_{\mathrm{j}} \mathrm{a}_{\mathrm{ij}} \\
\text { donde } \mathrm{a}_{\mathrm{ij}} \text { son los valores de la matriz de } \\
\text { adyacencia }\end{array}$ \\
\hline Intermediación & $\begin{array}{l}\text { Número de veces que un nodo (i) es } \\
\text { atravesado por los trayectos más cortos } \\
\text { de la red }\end{array}$ & $\begin{array}{l}\qquad \mathrm{C}_{\mathrm{B}}(\mathrm{i})=\sum_{\mathrm{j}, \mathrm{k}} \frac{\mathrm{b}_{\mathrm{jik}}}{\mathrm{b}_{\mathrm{jk}}} \\
\text { donde } \mathrm{b}_{\mathrm{jk}} \text { es el número de trayectos más } \\
\text { cortos del nodo } \mathrm{j} \text { al nodo } \mathrm{k}\end{array}$ \\
\hline Cercanía & $\begin{array}{l}\text { Medida de distancia entre un nodo (i) } \\
\text { y todos los demás nodos }\end{array}$ & $\begin{array}{l}\mathrm{C}_{\mathrm{C}}(\mathrm{i})=\sum_{\mathrm{j}=1}^{\mathrm{n}}(\mathrm{S})_{\mathrm{ij}} \\
\text { donde } \mathrm{S}_{\mathrm{ij}} \text { corresponden a la distancia } \\
\text { más corta del nodo i al nodo } \mathrm{j}\end{array}$ \\
\hline Vector propio & $\begin{array}{l}\text { Medida de la importancia o influencia } \\
\text { de un nodo (i) en la red }\end{array}$ & $\begin{array}{l}\quad \mathrm{x}_{\mathrm{i}}=\frac{1}{\lambda} \sum_{\mathrm{t} \in \mathrm{M}(\mathrm{i})} \mathrm{x}_{\mathrm{t}}=\frac{1}{\lambda} \sum_{\mathrm{t} \in \mathrm{G}} \mathrm{a}_{\mathrm{i}, \mathrm{t}} \mathrm{x}_{\mathrm{t}} \\
\text { donde } \mathrm{M}(\mathrm{i}) \text { es un conjunto de los } \\
\text { vecinos de } \mathrm{i}, \lambda \text { es una constante } \mathrm{y} \\
\mathrm{A}=\left(\mathrm{a}_{\mathrm{i}, \mathrm{t}}\right) \text { es la matriz de adyacencia }\end{array}$ \\
\hline
\end{tabular}

Fuente: ecuaciones extraídas de Freeman (1978) y Ruhnau (2000)

\subsection{Obtención de datos}

El comportamiento espacial de los visitantes en Billbao se ha recogido mediante dispositivos de localización GPS, más concretamente, registradores de datos (data loggers) GPS K-18U. El tipo de sensor utilizado en este dispositivo es un receptor de 50 canales de búsqueda, con código L1 C/A, dispone de una sensibilidad de adquisición y seguimiento de unos - $160 \mathrm{dBm}$ y emplea los Sistemas de Aumentación Basado en Satélites (SBAS) WAAS, EGNOS y MSAS. Los datos de localización proporcionados incluyen: longitud, latitud, altitud, hora, velocidad y dirección, así como las sentencias GPRMC y GPGGA correspondientes. Estas sentencias siguen el formato de datos standard NMEA, que hacen referencia a las especificaciones de comunicación entre dispositivos electrónicos y es la forma en la que tienen que transmitir los datos todos los fabricantes. Dependiendo de las prestaciones de estos dispositivos existen distintas sentencias NMEA, entre ellas las mencionadas GPRMC y GPGGA. 
Los dispositivos GPS se entregaron a los visitantes alojados en hoteles de categoría media- y fueron seleccionados entre los hoteles de Bilbao que estuvieron dispuestos a participar en este experimento. El proceso de recolección de datos fue realizado por CICtourGUNE (2011). El dispositivo GPS estaba programado para registrar su posición geográfica cada dos minutos. Como resultado, se obtuvo una muestra válida de 51 trackings, 51 turistas diferentes portando GPS. La investigación se llevó a cabo en los meses más importantes para el turismo cultural, concretamente en los meses de julio, agosto y septiembre de 2011, durante el período estival.

Por otro lado, los datos geográficos de la ciudad están basados en un mapa base de Esri, un proveedor de software SIG. Y finalmente, los datos de las atracciones culturales se obtuvieron de la base de datos Open Data Euskadi (Gobierno Vasco, 2012), una base de datos de atracciones o puntos de interés, de acceso abierto. Esta base de datos proporciona una descripción detallada de las atracciones culturales como museos, monumentos, etc., incluyendo las coordenadas geográficas. En cuanto al modelo de datos, la tabla de atracciones analizada tiene las siguientes variables principales: identificador del visitante, nombre de la atracción visitada, categoría (museo, monumento), marca de hora, latitud y longitud.

\subsection{Herramientas software}

Como base de datos se empleó PostgreSQL,tanto para los datos GPS como para la geolocalización de las atracciones. Y el análisis espacial se realizó mediante PostGIS. De este modo, el preprocesamiento y la detección de las atracciones visitadas se realizaron utilizando PostgreSQL / PostGIS y un script para la detección de los puntos de parada.

Los mapas (Figura 4 y Figura 5) están elaborados usando Leaflet for R (Cheng, Karambelkar \& Xie, 2017; Karambelkar \& Zheng, 2017), una librería JavaScript de código abierto para desarrollar mapas interactivos adaptada como un paquete de software R. El mapa base está formado por imágenes satelitales y fotografías aéreas de alta resolución proporcionadas por Esri. Así, se utilizaron los paquetes de R Leaflet y Leaflet.Extras para dibujar los puntos de calor y las atracciones en los mapas.

Las medidas de centralidad y la representación de la red se obtuvieron utilizando el software de análisis de redes Gephi (Bastian, Heymann \& Jacomy, 2009) y el plugin GeoLayout que muestra la red en base a atributos georeferenciados y proyecciones estándares. 


\section{Caso de estudio y resultados}

El estudio empírico se basa en el análisis de la movilidad de turistas en la ciudad de Bilbao, con el fin de identificar las atracciones centrales. Bilbao fue seleccionada por ser un ejemplo de regeneración urbana de una ciudad industrial, y una demostración de cómo un diseño arquitectónico e icónico, como el Museo Guggenheim Bilbao (MGB), puede ser utilizado como medio de desarrollo urbano (Gospodini, 2001; Plaza \& Haarich, 2009). Una muestra de su importancia es que el MGB atrae una media de 1000000 de visitantes al año.

A continuación, se presenta como se han obtenido los datos del caso de estudio, y en las secciones sucesivas se muestran los resultados de la aplicación de la metodología propuesta, obteniendo las atracciones más centrales de Bilbao. Por último, se realiza una comparación de esta clasificación de atracciones centrales resultante respecto a la clasificación que proporciona TripAdvisor.

\subsection{Extracción de las atracciones visitadas}

Tras el preprocesamiento de los datos GPS, se detectaron los puntos de parada aplicando el método propuesto. Una vez detectados los puntos de parada, la base de datos de atracciones se enlaza con la base de datos GPS, asociando una atracción a cada punto de parada, siempre y cuando el punto de parada se encuentre en el área de influencia de la atracción.

En este estudio se han detectado 213 visitas a más de veinte atracciones diferentes. De estas 213 visitas detectadas en Bilbao, 99 corresponden a atracciones culturales. Esta cifra se distribuye por categorías en 36 visitas a museos y 63 visitas a monumentos. Los atractivos culturales visitados, los que más destacan, se muestran en el mapa de Bilbao con el fin de alcanzar una mejor comprensión (ver Figura 4). Dos de las atracciones ("Museo del Pescador" y "Torre Salazar") no están en Bilbao sino en sus alrededores.

Con el fin de tener una visión general de la movilidad urbana y del consumo del espacio de los visitantes, se ha elaborado un mapa de calor de los datos de seguimiento GPS. Un mapa de calor es una representación gráfica de la densidad de puntos en un mapa, en nuestro caso la geolocalización de los visitantes. Leaflet $\mathrm{R}$ genera los mapas de calor asignando la densidad de cada píxel a partir de un valor directamente proporcional a la intensidad del punto (constante en este caso), e inversamente proporcional a la distancia entre el píxel y los puntos. En el mapa de calor representado en este documento, se ha aplicado un gradiente de color púrpura-amarillo. Hay 
que tener en cuenta que cuanto mayor sea la densidad, más claro será el color. Así, las atracciones culturales más visitadas están representadas por un color amarillo.

\section{Figura 4. Principales atracciones visitadas de Bilbao}

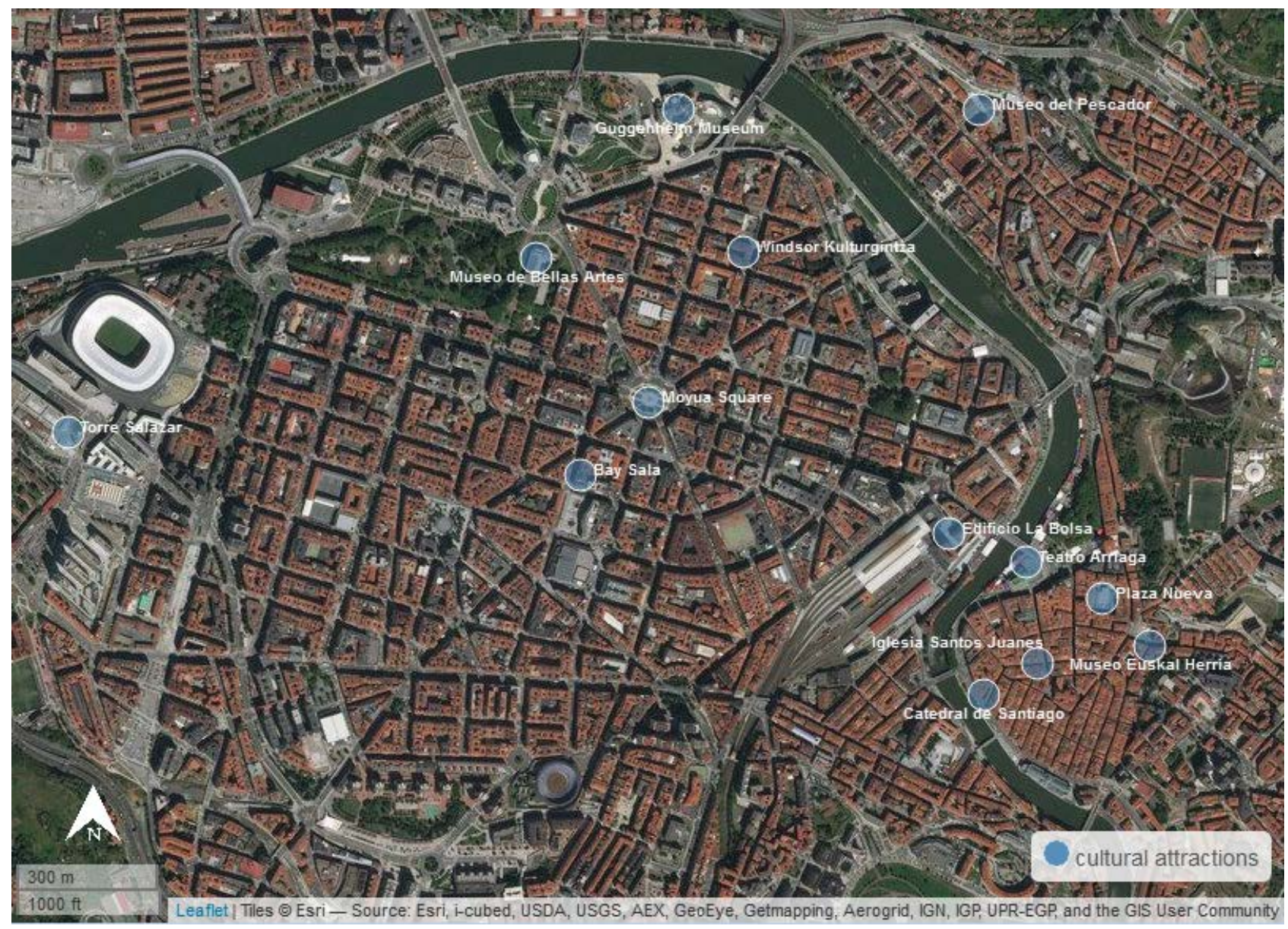

Fuente: elaboración propia

Como se puede ver en el mapa de calor de Bilbao (Figura 5), muchos de los puntos calientes están relacionados con la ubicación de las atracciones culturales. En el caso de Bilbao (ver Figura 4) las atracciones más populares se encuentran en el Casco Viejo y a lo largo de la ribera y la Gran Vía (Guggenheim, Bellas Artes, Plaza Moyua). Esto no es casualidad, ya que en Bilbao los lugares de interés cultural se encuentran principalmente en el Casco Viejo y en el centro de la ciudad. 
Figura 5. Consumo del espacio de los visitantes en el centro de Bilbao (datos GPS)

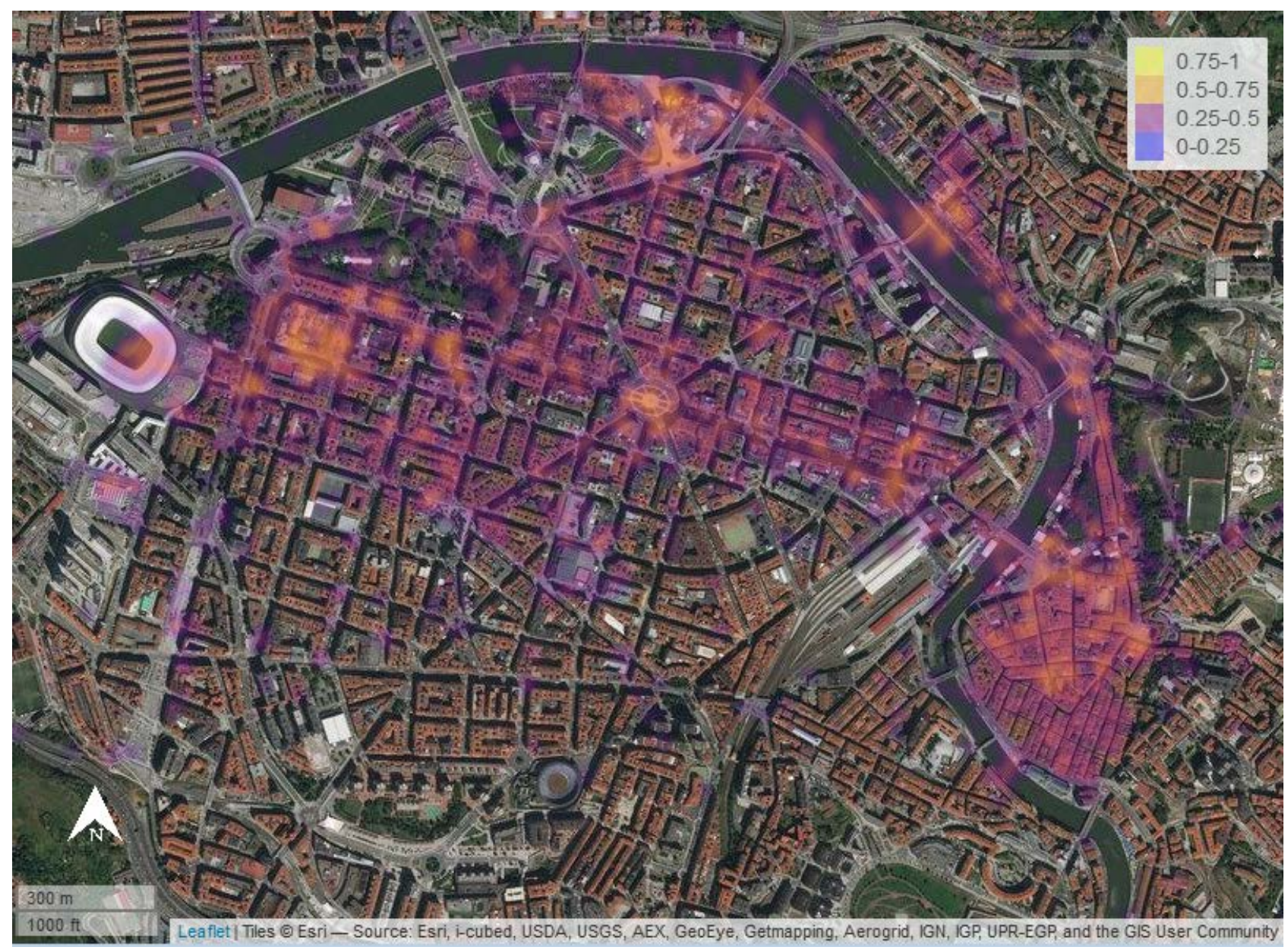

Fuente: elaboración propia

\subsection{Análisis de la red de atracciones visitadas: cuantificación de la centralidad}

Con el fin de hacer un seguimiento de la centralidad de las atracciones culturales visitadas, se llevó a cabo un análisis de la red, del cual se obtuvo la Figura 6. Hay que tener en cuenta que el tamaño de los nodos es proporcional a la medida de centralidad de grado, es decir, al número de conexiones de cada nodo. Por otro lado, el color representa el valor de centralidad de intermediación de cada nodo. Por lo tanto, en relación a la red trazada se puede decir que la atracción más popular y céntrica de Bilbao se encuentra en el Casco Viejo, donde se puede encontrar atracciones como la "Plaza Nueva", la "Catedral de Santiago", la Iglesia de "Santos Juanes" y el "Museo Vasco". Sin embargo, como el Casco Viejo puede considerarse una experiencia turística conjunta por sí misma, se ha tratado como una atracción única para realizar el análisis de la red y cuantificar su centralidad (ver Figura 7). 


\section{Figura 6. Red de las atracciones más visitadas en Bilbao}

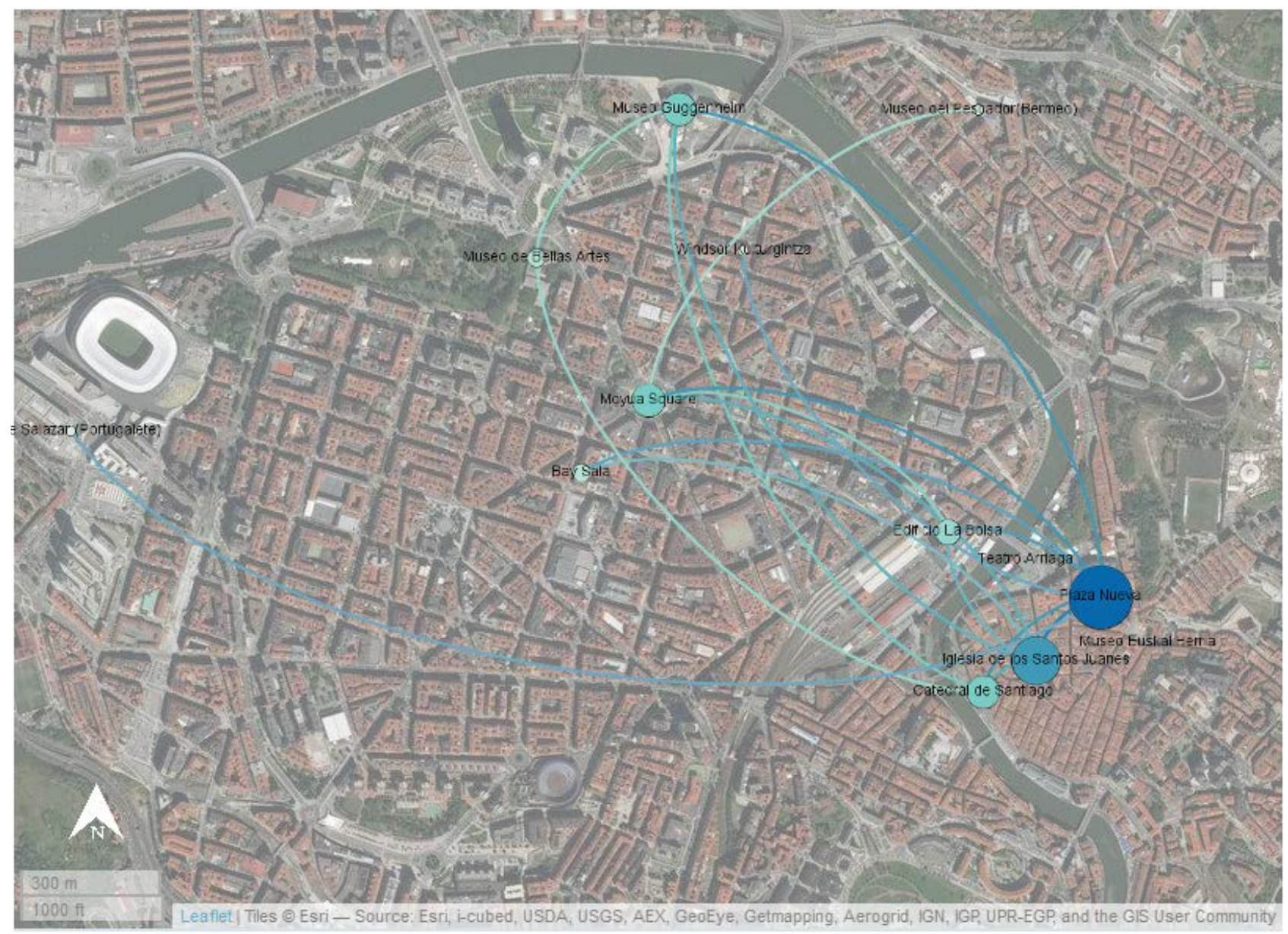

Fuente: elaboración propia

Los parámetros de red calculados se muestran en la Tabla 2. Los resultados sugieren la importancia de algunas atracciones, las más visitadas y las más interconectadas que otras con atracciones diferentes. Esta metodología puede ser fácilmente adoptada por los gestores de las ciudades para comprender mejor el comportamiento espacial de los visitantes y su impacto en las economías urbanas.

En cuanto a la centralidad de grado, en el caso de Bilbao, el Casco Viejo tiene el grado más alto (70 sobre 100). Esto significa que el 70 \% de los visitantes que visitan el Casco Viejo también visitan el $70 \%$ de las atracciones restantes. Como cualquier grado con un valor mayor de 20 se puede considerar una atracción interesante, ya que estará conectada al menos con un 20 \% de los nodos de la red, las atracciones a considerar en Bilbao serían: n2, n4, n11, n10, n7.

En cuanto a la centralidad de cercanía, hay que tener en cuenta que no sólo se incluye los vínculos directos de un nodo sino todos los vínculos indirectos con los demás nodos de la red. Así, no es 
de extrañar que los nodos tengan un índice de cercanía bastante similar, ya que el centro de Bilbao no es un territorio extenso, en el que incluso las distancias a pie son relativamente cortas.

Tabla 2. Medidas de centralidad de las atracciones visitadas en Bilbao

\begin{tabular}{|l|l|l|l|l|}
\hline Atracciones & Grado & Cercanía & Intermediación & Eigenvector \\
\hline Casco Viejo (n2) & 70.00 & 58.80 & 53.30 & 1.000 \\
\hline Plaza Moyua (n4) & 30.00 & 47.60 & 15.60 & 0.561 \\
\hline Edificio La Bolsa (n11) & 20.00 & 45.50 & 0.00 & 0.515 \\
\hline Museo Guggenheim (n10) & 20.00 & 43.50 & 0.00 & 0.489 \\
\hline Museo de Bellas Artes (n7) & 20.00 & 43.50 & 0.00 & 0.489 \\
\hline Bay Sala (n1) & 10.00 & 41.70 & 0.00 & 0.329 \\
\hline Torre Salazar (n14) & 10.00 & 41.70 & 0.00 & 0.329 \\
\hline Windsor Kulturgintza (n15) & 10.00 & 41.70 & 0.00 & 0.329 \\
\hline Museo del Pescador (n8) & 10.00 & 35.70 & 0.00 & 0.186 \\
\hline
\end{tabular}

Fuente: elaboración propia

Figura 7. Red de las atracciones más visitadas en Bilbao, incluyendo el Casco Antiguo

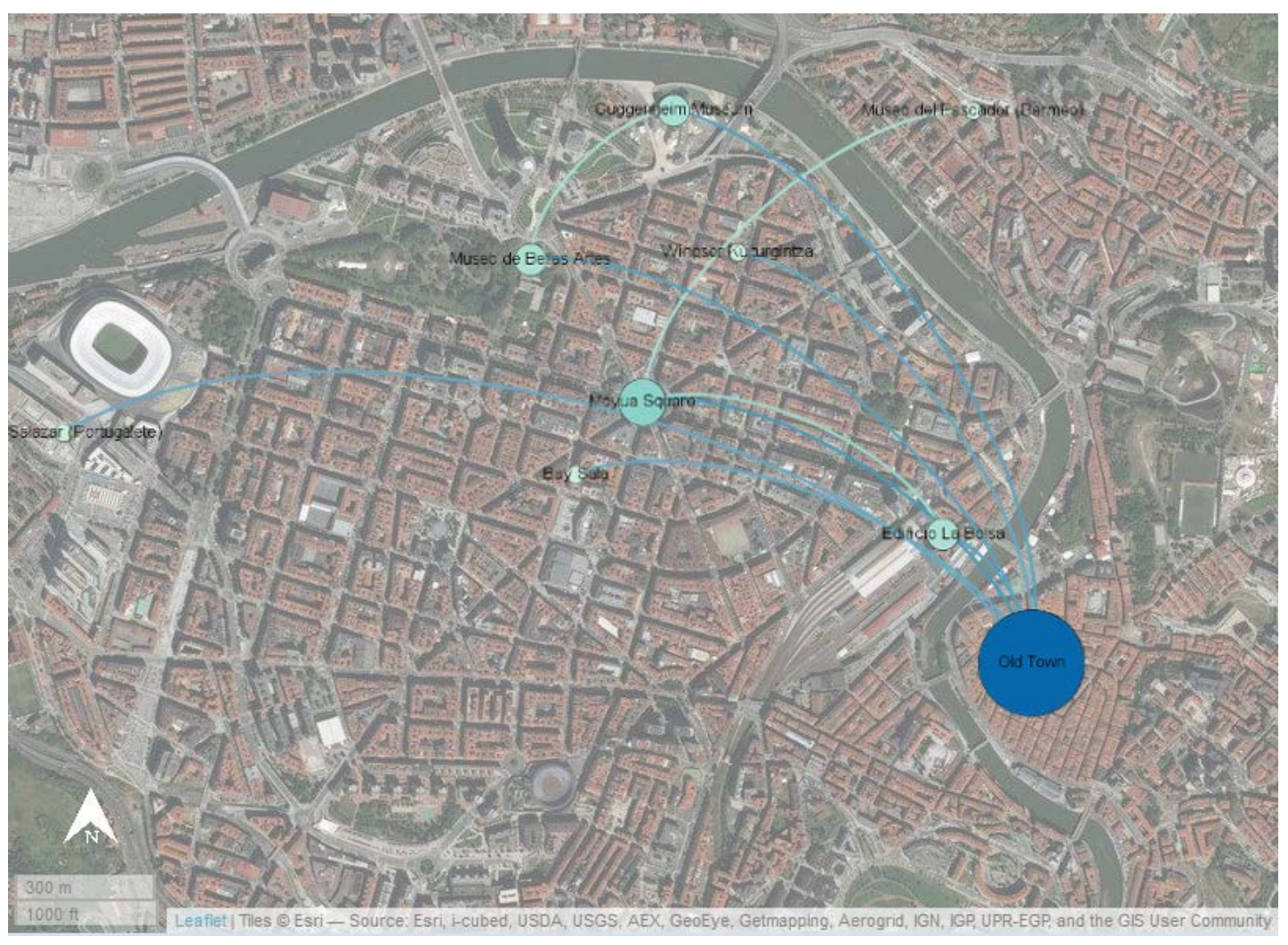

Fuente: elaboración propia 
En relación a la centralidad de intermediación (ver Tabla 1), los resultados son los siguientes. En el caso de Bilbao, el Casco Viejo es el que presenta el mayor número del índice de intermediación (53,3 sobre 100). Esto significa que el $53 \%$ de los visitantes (que toman el camino más corto) pasan por el Casco Viejo en algún momento. Cualquier valor de la intermediación superior a 0 se puede considerar una atracción interesante, como es el caso de la Plaza Moyua (n4) de Bilbao. Por lo tanto, estos nodos se encuentran en el camino más corto entre todos los pares de nodos. En cierto modo, la centralidad de intermediación está midiendo la accesibilidad de los nodos teniendo en cuenta todas esas rutas.

Teniendo en cuenta el objetivo principal de esta investigación, el principal índice con el que medir la centralidad de las atracciones culturales es el vector propio o eigenvector. El eigenvector refleja la importancia de las conexiones y es indicativo de la influencia del nodo. En cuanto a los valores del vector propio, se debe tener en cuenta cualquier atracción con un valor mayor o similar a 0,5. Así pues, según los valores del eigenvector, las atracciones más importantes de Bilbao son: Casco Viejo (n1), Plaza Moyua (n4), Edificio la Bolsa (n11), Museo Guggenheim (n10) y Museo "Bellas Artes" (n7). Esto significa que, además de ser los nodos más importantes, también están bien interconectados. Por lo tanto, los nodos destacados anteriormente deben ser considerados como las atracciones centrales de Bilbao.

Otra medida interesante es la longitud media de trayecto (average path length), una medida de toda la red y no una medida de un único nodo. La longitud media de trayecto muestra lo compacta que es la red y cuantifica el número medio de nodos necesarios a lo largo de los trayectos más cortos para conectar cualquier par de nodos. La longitud media del trayecto es bastante pequeña, de hecho en el caso de Bilbao es de 1,86 arcos. Esto significa que las atracciones culturales se concentran en una pequeña área y más concretamente en el centro de la ciudad. Esto también puede reflejar la alta transitabilidad de los espacios consumidos e implica a su vez que los destinos pueden ser visitados fácilmente.

\subsection{Comparando los resultados con TripAdvisor}

El Museo Guggenheim Bilbao es la atracción cultural "imprescindible" a visitar en Bilbao para casi todos los turistas, y como tal, el Guggenheim es el activo turístico más destacado de TripAdvisor con 13.095 opiniones, seguido muy por detrás de la Plaza Nueva en el Casco Viejo con 2783 opiniones (ver Tabla 3). 
Tabla 3. Ranking de atracciones (Bilbao) de TripAdvisor basado en el número de opiniones

\begin{tabular}{|l|r|}
\hline \multicolumn{1}{|c|}{ Atracciones } & \multicolumn{1}{|c|}{$\begin{array}{c}\text { Número de } \\
\text { Opiniones }\end{array}$} \\
\hline Museo Guggenheim & 13,095 \\
\hline Plaza Nueva & 2,783 \\
\hline Museo de Bellas Artes & 1,992 \\
\hline Plaza Moyua & 641 \\
\hline Catedral de Santiago & 259 \\
\hline Museo Euskal Herria & 216 \\
\hline Teatro Arriaga & 134 \\
\hline Torre Salazar & 42 \\
\hline Museo del Pescador & 41 \\
\hline Iglesia Santos Juanes & 2 \\
\hline Edificio La Bolsa & 0 \\
\hline Bay Sala & 0 \\
\hline Windsor Kulturgintza & \\
\hline TrinA & \\
\hline
\end{tabular}

Fuente: TripAdvisor.com (consultada el 14 de marzo de 2018).

Nuestros resultados, sin embargo, señalan que no es el Museo Guggenheim el bien cultural más visitado de Bilbao, sino el Casco Viejo. En este sentido, la utilización única de datos del estilo de TripAdvisor puede llevar a conclusiones engañosas a la hora de considerar las atracciones más populares como las más consumidas en los destinos turísticos (Kladou \& Mavragani, 2015). En otras palabras, la metodología propuesta en este artículo y que tiene en consideración el consumo espacial de los visitantes, aporta valor al estado del arte y es complementario a otras fuentes de datos.

\section{Conclusiones}

La metodología utilizada en este estudio conduce a resultados inesperados pero significativos: Mientras que los contenidos de las redes sociales (por ejemplo, TripAdvisor) y los expertos (agentes de turismo) señalan al Guggenheim como el principal activo turístico, nuestro método destaca el Casco Viejo como el lugar más visitado de Bilbao. Este hecho sugiere que existe una 
diferencia entre el comportamiento espacial real de los visitantes y su comportamiento virtual en redes sociales como TripAdvisor. Por lo tanto, esta metodología tiene el potencial de proporcionar información valiosa para los gestores de la ciudad.

Cabe mencionar también que la geoinformación aplicada en esta metodología es clave ya que las atracciones visitadas se obtienen a partir de datos geolocalizados, tanto en el caso de los visitantes como de las atracciones. De la misma manera, la visualización de los datos es complementaria a la metodología, ya que es útil para obtener una mejor comprensión de los resultados. Además, las herramientas de visualización aplicadas en este estudio son de código abierto y software libre (paquetes R, Gephi, PostgreSQL / PostGIS), por lo que pueden ser utilizadas por cualquier persona que realice tareas similares a las que se incluyen en este trabajo.

En relación al tipo de redes utilizado hay que destacar que las redes unidireccionales y no ponderadas han cumplido con el objetivo marcado de realizar el análisis topológico de la red de atracciones. En cualquier caso este tipo redes tiene sus limitaciones y en futuras investigaciones convendría sopesar el uso de redes ponderadas y/o direccionales, con el objeto de realizar el análisis de flujos y afluencia turística.

Así, las investigaciones futuras deben tener en cuenta varios aspectos. Por un lado, convendría realizar un análisis ponderado de la red, de modo que el número de visitantes pueda ser tratado como ponderaciones de los enlaces. Para que este análisis fuera significativo sería necesario aumentar el número de turistas de la muestra analizada. Además, para lograr una mejor comprensión de la movilidad, se debe considerar la secuencia de visitas de los visitantes, realizando un análisis dirigido de la red. Del mismo modo, sería interesante poner en evidencia los caminos o trayectos entre atracciones. De esta manera, los planificadores podrían saber cuáles son las calles más utilizadas.

Además, en un futuro cercano convendría actualizar los datos de movilidad que se disponen, ya que fueron obtenidos en el verano de 2011. Una vez obtenidos los nuevos datos el objetivo sería realizar una comparación de los resultados y comprobar si las conclusiones se mantienen en el tiempo.

Por último, como esta metodología puede ser fácilmente adoptada por otras ciudades, sería interesante realizar un estudio más amplio con otras ciudades, con el fin de obtener conclusiones generalizables. Estos nuevos conocimientos deberían ayudar a los gestores urbanos a identificar mejor las atracciones centrales, comprender la movilidad de los visitantes y evaluar su impacto en la economía urbana. 
Agradecimientos: Las autoras agradecen la ayuda económica aportada por el Ministerio de Economía y Empresa (MINECOR/FEDER 2015 CREA-NETWORK CSO2015-65265-C4-3-R).

Declaración responsable: Las/os autoras/es declaran que no existe ningún conflicto de interés con relación a la publicación de este artículo. Las tareas se han distribuido de la siguiente manera: Las/os tres autoras/es han participado en la revisión bibliográfica, el diseño de la metodología y la redacción del artículo. La realización del análisis cuantitativo ha estado a cargo de I. Aranburu. 


\section{Bibliografía}

Abedi, N., Bhaskar, A., \& Chung, E. (2014). Tracking spatio-temporal movement of human in terms of space utilization using Media-Access-Control address data. Applied Geography, 51, 7281. https://doi.org/10.1016/j.apgeog.2014.04.001

Aranburu, I., Plaza, B., \& Esteban, M. (2016). Sustainable Cultural Tourism in Urban Destinations: Does Space Matter? Sustainability, 8(8), 699. https://doi.org/10.3390/su8080699

Ashbrook, D., \& Starner, T. (2003). Using GPS to learn significant locations and predict movement across multiple users. Personal and Ubiquitous Computing, 7(5), 275286. https://doi.org/10.1007/s00779-003-0240-0

Axhausen, K. W., Schonfelder, S., Wolf, J., Oliveira, M., \& Samaga, U. (2003). Eighty weeks of GPS traces: Approaches to enriching trip information. In The 83rd Transportation Research Board Meeting (pp. 1870-1876). Washington, DC.

Barthélemy, M. (2011). Spatial networks. Physics Reports, 499(1-3), 1101. https://doi.org/10.1016/j.physrep.2010.11.002

Bastian, M., Heymann, S., \& Jacomy, M. (2009). Gephi: An Open Source Software for Exploring and Manipulating Netrieved from http://www.aaai.org/ocs/index.php/ICWSM/09/paper/view/154

Bohte, W., \& Maat, K. (2009). Deriving and validating trip purposes and travel modes for multi-day GPS-based travel surveys: A large-scale application in the Netherlands. Transportation Research Part C: Emerging Technologies, 17(3), 285-297. https://doi.org/10.1016/j.trc.2008.11.004

Capello, R., \& Perucca, G. (2017). Cultural Capital and Local Development Nexus: Does the Local Environment Matter? In Socioeconomic Environmental Policies and Evaluations in Regional Science (pp. 103-124). Springer. https://doi.org/10.1007/978-981-10-0099-7_6

Cheng, J., Karambelkar, B., \& Xie, Y. (2017). Leaflet: Create Interactive Web Maps with the JavaScript "Leaflet" Library. R package version 1.1.0. Retrieved from https://cran.rproject.org/package=leaflet

CICtourGUNE. (2011). Centro de Investigación Cooperativa en Turismo. Spain.

Demšar, U., Špatenková, O., \& Virrantaus, K. (2008). Identifying Critical Locations in a Spatial Network with Graph Theory. Transactions in GIS, 12(1), 61-82. https://doi.org/10.1111/j.14679671.2008.01086.x 
Dietvorst, A., \& Ashworth, G. (1995). Tourist behaviour and the importance of time-space analysis. In Tourism and spatial transformations. (pp. 163-181). CAB International.

Esteban, M. (1999). Bilbao, luces y sombras del titanio: el proceso de regeneración del Bilbao metropolitano. Servicio Editorial de la Universidad del País Vasco.

Freeman, L. C. (1978). Centrality in social networks conceptual clarification. Social Networks, 1(3), 215-239. https://doi.org/10.1016/0378-8733(78)90021-7

Freytag, T. (2002). Tourism in Heidelberg: getting a picture of the city and its visitors. In City tourism 2002: Proceedings of European Cities Tourism's International Conference in Vienna, Austria, 2002 (pp. 211-219). Springer-Verlag Wien.

Fu, Z., Tian, Z., Xu, Y., \& Qiao, C. (2016). A Two-Step Clustering Approach to Extract Locations from Individual GPS Trajectory Data. ISPRS International Journal of Geo-Information, 5(10), 166. https://doi.org/10.3390/ijgi5100166

Galí Espelt, N., \& Donaire Benito, J. A. (2018). First-time versus repeat visitors' behavior patterns: a GPS analysis. Boletín de La Asociación de Geógrafos Españoles, (78), 2711. https://doi.org/10.21138/bage.2711

García-Palomares, J. C., Gutiérrez, J., \& Mínguez, C. (2015). Identification of tourist hot spots based on social networks: A comparative analysis of European metropolises using photo-sharing services and GIS. Applied Geography, 63, 408417. https://doi.org/10.1016/j.apgeog.2015.08.002

Gobierno Vasco. (2012). Open Data Euskadi. Retrieved from http://opendata.euskadi.eus

Gospodini, A. (2001). Urban Design, Urban Space Morphology, Urban Tourism

New Paradigm Concerning. European Planning Studies, 9(7), 925-

\section{4. https://doi.org/10.1080/0965431012007984}

Grinberger, A. Y., Shoval, N., \& McKercher, B. (2014). Typologies of tourists' time-space consumption: a new approach using GPS data and GIS tools. Tourism Geographies, 16(1), 105123. https://doi.org/10.1080/14616688.2013.869249

Gschwender, A., Munizaga, M., \& Simonetti, C. (2016). Using smart card and GPS data for policy and planning: The case of Transantiago. Research in Transportation Economics, 59, 242249. https://doi.org/10.1016/j.retrec.2016.05.004

Hall, C. M., \& Ram, Y. (2019). Measuring the relationship between tourism and walkability? Walk 
Score and English tourist attractions. Journal of Sustainable Tourism, 27(2), 223240. https://doi.org/10.1080/09669582.2017.1404607

Hartman, G. W. (1950). The Central Business District - A Study in Urban Geography. Economic Geography, 26(4), 237. https://doi.org/10.2307/141260

Hasan, S., Schneider, C. M., Ukkusuri, S. V, \& González, M. C. (2013). Spatiotemporal Patterns of Urban Human Mobility. Journal of Statistical Physics, 151(1-2), 304318. https://doi.org/10.1007/s10955-012-0645-0

Hawelka, B., Sitko, I., Beinat, E., Sobolevsky, S., Kazakopoulos, P., \& Ratti, C. (2014). Geo-located Twitter as proxy for global mobility patterns. Cartography and Geographic Information Science, 41(3), 260-271. https://doi.org/10.1080/15230406.2014.890072

Jayasinghe, A., Sano, K., \& Rattanaporn, K. (2017). Application for developing countries: Estimating trip attraction in urban zones based on centrality. Journal of Traffic and Transportation Engineering (English Edition), 4(5), 464-476.

Kaplan, E. D. (1996). Understanding GPS: Principles and Applications. Artech House.

Karambelkar, B., \& Zheng, B. (2017). Extra Functionality for "leaflet" Package. Retrieved from https://cran.r-project.org/package=leaflet.extras

Kladou, S., \& Mavragani, E. (2015). Assessing destination image: An online marketing approach and the case of TripAdvisor. Journal of Destination Marketing \& Management, 4(3), 187193. https://doi.org/10.1016/j.jdmm.2015.04.003

Kourtit, K., Nijkamp, P., \& Partridge, M. D. (2013). The New Urban World. European Planning Studies, 21(3), 285-290. https://doi.org/10.1080/09654313.2012.716242

Lau, G., \& McKercher, B. (2006). Understanding tourist movement patterns in a destination: A GIS approach. Tourism and Hospitality Research, 7(1), 3949. https://doi.org/10.1057/palgrave.thr.6050027

Le-Klähn, D.-T. (2016). Sustainable Tourist Mobility: Implications for Urban Destination Management. In Sustainable Mobility in Metropolitan Regions (pp. 55-63). Wiesbaden: Springer Fachmedien Wiesbaden. https://doi.org/10.1007/978-3-658-14428-9_4

Leung, X. Y., Wang, F., Wu, B., Bai, B., Stahura, K. A., \& Xie, Z. (2012). A Social Network Analysis of Overseas Tourist Movement Patterns in Beijing: the Impact of the Olympic Games. International Journal of Tourism Research, 14(5), 469-484. https://doi.org/10.1002/jtr.876 
Lew, A., \& McKercher, B. (2006). Modeling Tourist Movements. A Local Destination Analysis. Annals of Tourism Research, 33(2), 403-423. https://doi.org/10.1016/j.annals.2005.12.002

Liu, B., Huang, S. S., \& Fu, H. (2017). An application of network analysis on tourist attractions: The case of Xinjiang, China. Tourism Management, 58, 132141. https://doi.org/10.1016/j.tourman.2016.10.009

Mazimpaka, J. D., \& Timpf, S. (2015). Exploring the Potential of Combining Taxi GPS and Flickr Data for Discovering Functional Regions. In AGILE 2015 (pp. 3-18). Springer. https://doi.org/10.1007/978-3-319-16787-9_1

Mckercher, B., \& Lau, G. (2008). Movement Patterns of Tourists within a Destination. Tourism Geographies, 10(3), 355-374. https://doi.org/10.1080/14616680802236352

McKercher, B., Shoval, N., Ng, E., \& Birenboim, A. (2012). First and Repeat Visitor Behaviour: GPS Tracking and GIS Analysis in Hong Kong. Tourism Geographies, 14(1), 147161. https://doi.org/10.1080/14616688.2011.598542

Modsching, M., Kramer, R., Hagen, K. Ten, \& Gretzel, U. (2008). Using Location-based Tracking Data to Analyze the Movements of City Tourists. Information Technology \& Tourism, 10(1), 3142. https://doi.org/10.3727/109830508785059011

Montoliu, R., Blom, J., \& Gatica-Perez, D. (2013). Discovering places of interest in everyday life from smartphone data. Multimedia Tools and Applications, 62(1), 179207. https://doi.org/10.1007/s11042-011-0982-z

Murakami, E., \& Wagner, D. P. (1999). Can using global positioning system (GPS) improve trip reporting? Transportation Research Part C: Emerging Technologies, 7(2), 149-165.

Page, S. (2004). Transport and tourism. In A companion to tourism. Blackwell Publishing, Malden, Mass.

Pearce, D. G. (1988). Tourist time-budget. Annals of Tourism Research, 15(1), 106-121.

Peng, X., \& Huang, Z. (2017). A Novel Popular Tourist Attraction Discovering Approach Based on Geo-Tagged Social Media Big Data. ISPRS International Journal of Geo-Information, 6(7), 216. https://doi.org/10.3390/ijgi6070216

Plaza, B., \& Haarich, S. N. (2009). Museums for urban regeneration? Exploring conditions for their effectiveness. Journal of Urban Regeneration and Renewal, 2(3), 259-271. Retrieved from https://www.ingentaconnect.com/content/hsp/jurr/2009/00000002/00000003/art00 
Quiroga, C. A., \& Bullock, D. (1998). Travel time studies with global positioning and geographic information systems: an integrated methodology. Transportation Research Part C: Emerging Technologies, 6(1), 101-127.

Ratti, C., Sobolevsky, S., Calabrese, F., Andris, C., Reades, J., Martino, M., „Strogatz, S. H. (2010). Redrawing the Map of Great Britain from a Network of Human Interactions. PLoS ONE, 5(12), e14248. hitps://doi.org/10.1371/journal.pone.0014248

Richards, G. (2010). Increasing the Attractiveness of Places Through Cultural Resources. Tourism Culture \& Communication, 10(1), 47-58.

https://doi.org/10.3727/109830410X12629765735678

Richards, G. (2011). Creativity and tourism. Annals of Tourism Research, 38(4), 12251253. https://doi.org/10.1016/j.annals.2011.07.008

Richards, G. (2014). Creativity and tourism in the city. Current Issues in Tourism, 17(2), 119144. https://doi.org/10.1080/13683500.2013.783794

Ruhnau, B. (2000). Eigenvector-centrality-a node-centrality? Social Networks, 22(4), 357365. https://doi.org/10.1016/S0378-8733(00)00031-9

Sacco, P., Ferilli, G., \& Blessi, G. T. (2014). Understanding culture-led local development: A critique of alternative theoretical explanations. Urban Studies, 51(13), 2806-2821.

Schönfelder, S., Axhausen, K. W., Antille, N., \& Bierlaire, M. (2002). Exploring the Potentials of Automatically Collected GPS Data for Travel Behaviour Analysis: A Swedish Data Source. Arbeitsberichte Verkehrs-Und Raumplanung, 124.

Schuessler, N., \& Axhausen, K. W. (2009). Identifying trips and activities and their characteristics from GPS raw data without further information. Transportation Research Record: Journal of the Transportation Research Board., 2105, 1-28.

Scott, J. P. (2000). Social Network Analysis: A Handbook. SAGE Publications. Retrieved from hittps://uk.sagepub.com/en-gb/eur/the-sage-handbook-of-social-networkanalysis/book232753

Shaw, G., Agarwal, S., \& Bull, P. (2000). Tourism consumption and tourist behaviour: A British perspective. Tourism Geographies, 264289. https://doi.org/10.1080/14616680050082526 
Shen, L., \& Stopher, P. R. (2014). Review of GPS Travel Survey and GPS Data-Processing Methods. Transport Reviews, 34(3), 316-334.https://doi.org/10.1080/01441647.2014.903530 Shoval, N., \& Isaacson, M. (2009). Tourist Mobility and Advanced Tracking Technologies. Routledge Advances in Tourism series.

Shoval, N., McKercher, B., Ng, E., \& Birenboim, A. (2011). Hotel location and tourist activity in cities. Annals of Tourism Research, 38(4), 15941612. https://doi.org/10.1016/j.annals.2011.02.007

Silberberg, T. (1995). Cultural Tourism and Business Opportunities for Museums and Heritage Sites. Tourism Management, 16(5), 361-365. https://doi.org/10.1016/0261-5177(95)00039-Q

Soh, H., Lim, S., Zhang, T., Fu, X., Lee, G. K. K., Hung, T. G. G., I Wong, L. (2010). Weighted complex network analysis of travel routes on the Singapore public transportation system. Physica A: Statistical Mechanics and Its Applications, 389(24), 58525863. https://doi.org/10.1016/j.physa.2010.08.015

Stienmetz, J. L., \& Fesenmaier, D. R. (2015). Estimating value in Baltimore, Maryland: An attractions network analysis. Tourism Management, 50, 238252. https://doi.org/10.1016/j.tourman.2015.01.031

Stopher, P., Jiang, Q., \& FitzGerald, C. (2005). Processing GPS data from travel surveys. In 2nd International Collogium on the behavioural foundations of integrated land-use and transportation models: frameworks, models and applications (pp. 1-21). Toronto.

Taczanowska, K., González, L.-M., Garcia-Massó, X., Muhar, A., Brandenburg, C., \& TocaHerrera, J.-L. (2014). Evaluating the structure and use of hiking trails in recreational areas using a mixed GPS tracking and graph theory approach. Applied Geography, 55, 184192. https://doi.org/10.1016/j.apgeog.2014.09.011

Tchetchik, a., Fleischer, A., \& Shoval, N. (2009). Segmentation of Visitors to a Heritage Site Using High-resolution Time-space Data. Journal of Travel Research, 48(2), 216229. https://doi.org/10.1177/0047287509332307

Throsby, D. (2017). Culturally sustainable development: theoretical concept or practical policy instrument? International Journal of Cultural Policy, 23(2), 133-147.

Timmermans, H., Arentze, T., \& Joh, C.-H. (2002). Analysing space-time behaviour: new approaches to old problems. Progress in Human Geography, 26(2), 175190. https://doi.org/10.1191/0309132502ph363ra 
Urtasun, A., \& Gutiérrez, I. (2006). Tourism agglomeration and its impact on social welfare: An empirical approach to the Spanish case. Tourism Management, 27(5), 901-912.

Wang, D., Pedreschi, D., Song, C., Giannotti, F., \& Barabasi, A.-L. (2011). Human mobility, social ties, and link prediction. In Proceedings of the 17th ACM SIGKDD international conference on Knowledge discovery and data mining - KDD'11 (p. 1100). New York, New York, USA: ACM Press. https://doi.org/10.1145/2020408.2020581

Wolf, J., Guensler, R., \& Bachman, W. (2001). Elimination of the travel diary: Experiment to derive trip purpose from global positioning system travel data. Transportation Research Record: Journal of the Transportation Research Board, 1768(1), 125-134.

Xiang, L., Gao, M., \& Wu, T. (2016). Extracting Stops from Noisy Trajectories: A Sequence Oriented Clustering Approach. ISPRS International Journal of Geo-Information, 5(3), 29. https://doi.org/10.3390/ijgi5030029

Yu, W., Ai, T., \& Shao, S. (2015). The analysis and delimitation of Central Business District using network kernel density estimation. Journal of Transport Geography, 45, 32-47.

Yuan, J., Zheng, Y., \& Xie, X. (2012). Discovering regions of different functions in a city using human mobility and POls. In Proceedings of the 18th ACM SIGKDD international conference on Knowledge discovery and data mining (pp. 186-194). New York, USA: ACM Press. https://doi.org/10.1145/2339530.2339561

Zhong, C., Schläpfer, M., Müller Arisona, S., Batty, M., Ratti, C., \& Schmitt, G. (2017). Revealing centrality in the spatial structure of cities from human activity patterns. Urban Studies, 54(2), 437455. https://doi.org/10.1177/0042098015601599

Zornoza Gallego, C., \& Salom Carrasco, J. (2018). Geolocalized Tweets for assessing daily mobility: methodology to analyse and detect homelocation in the urban area of Valencia. Boletín de La Asociación de Geógrafos Españoles, (79). https://doi.org/10.21138/bage.2464 